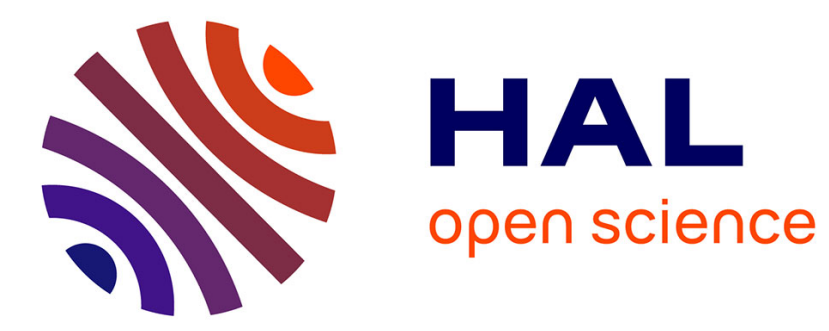

\title{
Extraordinary Hall effect based magnetic logic applications
}

T. Liu, D. Lacour, F. Montaigne, S. Le Gall, M. Hehn, Thomas Hauet

\section{To cite this version:}

T. Liu, D. Lacour, F. Montaigne, S. Le Gall, M. Hehn, et al.. Extraordinary Hall effect based magnetic logic applications. Applied Physics Letters, 2015, 106 (5), pp.052406. 10.1063/1.4907615 . hal-01246637

\section{HAL Id: hal-01246637 https://hal.science/hal-01246637}

Submitted on 12 Mar 2020

HAL is a multi-disciplinary open access archive for the deposit and dissemination of scientific research documents, whether they are published or not. The documents may come from teaching and research institutions in France or abroad, or from public or private research centers.
L'archive ouverte pluridisciplinaire HAL, est destinée au dépôt et à la diffusion de documents scientifiques de niveau recherche, publiés ou non, émanant des établissements d'enseignement et de recherche français ou étrangers, des laboratoires publics ou privés. 


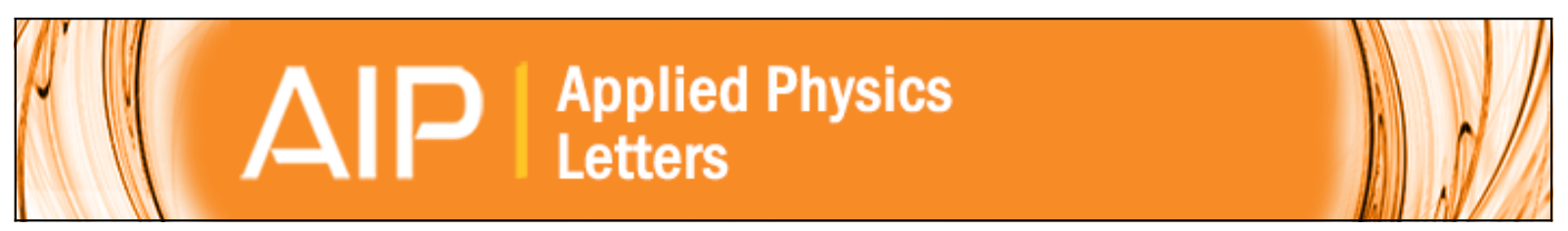

\section{Extraordinary Hall effect based magnetic logic applications}

T. Liu, D. Lacour, F. Montaigne, S. Le Gall, M. Hehn, and T. Hauet

Citation: Applied Physics Letters 106, 052406 (2015); doi: 10.1063/1.4907615

View online: http://dx.doi.org/10.1063/1.4907615

View Table of Contents: http://scitation.aip.org/content/aip/journal/apl/106/5?ver=pdfcov

Published by the AIP Publishing

\section{Articles you may be interested in}

Compensation of orange-peel coupling effect in magnetic tunnel junction free layer via shape engineering for nanomagnet logic applications

J. Appl. Phys. 115, 17B902 (2014); 10.1063/1.4863935

Compact modeling of perpendicular nanomagnetic logic based on threshold gates

J. Appl. Phys. 115, 17D104 (2014); 10.1063/1.4857555

Magnetoresistive effects in perpendicularly magnetized Tb-Co alloy based thin films and spin valves

J. Appl. Phys. 111, 083904 (2012); 10.1063/1.3703666

Writing and erasing data in magnetic domain wall logic systems

J. Appl. Phys. 100, 123908 (2006); 10.1063/1.2402026

Programmable spintronics logic device based on a magnetic tunnel junction element

J. Appl. Phys. 97, 10D509 (2005); 10.1063/1.1857655

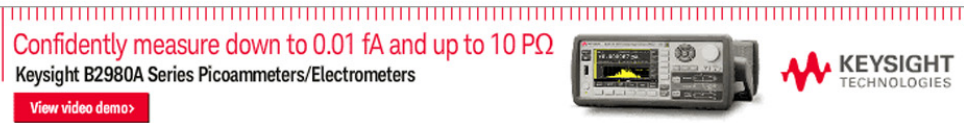




\title{
Extraordinary Hall effect based magnetic logic applications
}

\author{
T. Liu, D. Lacour, F. Montaigne, S. Le Gall, ${ }^{a)}$ M. Hehn, and T. Hauet \\ Institut Jean Lamour, UMR CNRS-Université de Lorraine, 54506 Vandoeuvre lès Nancy, France
}

(Received 6 November 2014; accepted 25 January 2015; published online 5 February 2015)

\begin{abstract}
Extraordinary Hall Effect (EHE) based original concepts of a reconfigurable logic gate and a multibit logic comparator are presented. They exploit the EHE voltage that develops on cross cells connected in series that has no size limitation down to the nanometer scale. Experimental demonstrations are performed on both micro- and nanometer lateral size crosses made of ferrimagnetic TbCo alloy. The simplicity of the device architecture and its robustness make it advantageous when compared with existing systems. (C) 2015 AIP Publishing LLC. [http://dx.doi.org/10.1063/1.4907615]
\end{abstract}

In many security application fields, including secure microcontrollers, subscriber identity module (SIM) cards, banking cards, biometric authentication chips, and near field communication, a logic operation of comparing the user inputs with a reference code needs to be carried out. In the context of the conventional flash technology, the reference codes are usually stored in a recording device and are extracted and injected into the logic circuit during the comparison process. This extraction process not only causes time and energy wasting but also results in a security risk of possible exposure of reference codes to a malicious user. Recently, a new concept was proposed based on series of connected Magnetic Tunnel Junctions (MTJs). ${ }^{1}$ In this design, the reference and free layers of the MTJ cells are naturally antiferromagnetically coupled such that the resistance of the MTJs connected in series is maximum. The digital test code is compared to the reference by injecting currents into metal wires settled under the MTJs. Each wire corresponds to a bit, whose level is encoded in the sense of the current. If the code matches, the current is sent in a sense such that the Oersted field reinforces the antiferromagnetic coupling and the MTJs total resistance remains high. In the opposite case, the Oersted field aligns the two MTJ electrode magnetizations, and the total resistance drops and an error in the code is detected. As the logic comparison is carried out on chip, where the reference code is located, the manipulation time and energy consumption are reduced. The risk of possible exposure of the reference codes is suppressed.

This comparator device is one example among the many proposed logic devices based on magnetic spin valves (SVs) or MTJs. ${ }^{1-4}$ SVs and MTJs are already widely used in industry as sensors ${ }^{5,6}$ and their properties will continue to be improved since they are considered for the next generation of spintronic applications, e.g., spin-transfer torque magnetic random access memory (STT-MRAM) ${ }^{7-9}$ and spin-transfer nano-oscillators (STNOs). ${ }^{10-12}$ However, those spintronic devices are rather complex from the material and the micro and nanoelectronic process point of view which may affect cost and reproducibility. From the material science view side, one can observe the complexity of the SVs or MTJs stacking as well as the drastic reduction of layer thicknesses

\footnotetext{
${ }^{\text {a) }}$ Current address: Génie Electrique et Electronique de Paris, UMR-CNRS 8507, SUPELEC, Univ. Paris-Sud XI, UPMC Paris VI, 91192, Gif-surYvette, France.
}

down to the atomic level. ${ }^{13}$ For many logic applications, such complexity is unnecessary and one may turn back toward simpler architecture.

In the present work, we propose and experimentally demonstrate two original concepts of magnetic logic devices based on the extraordinary Hall effect $(\mathrm{EHE})^{14,15}$ which have strong potential for logic applications because of their simplicity and scalability. ${ }^{16,17}$ The first one is a programmable Exclusive OR (XOR) or Exclusive NOR (XNOR) logic gate and the second one is a logic multi-bit comparator. Both architectures are based on EHE crosses connected in series. Compared with the similar concepts based on the other architectures, ${ }^{1,2}$ the two proposed logic devices possess much simpler structure with a single magnetic film and has large EHE signals with high signal-to-noise ratio.

The architectures of the XOR and XNOR logic gates are presented in Fig. 1(a). They are composed of two electrically connected crosses. Two input currents $\left(I_{1}\right.$ and $\left.I_{2}\right)$ are directly applied on the transverse arms of the two magnetized crosses. The logic computation is made simply by measuring the Hall voltage along the direction of common longitudinal arm $\left(V_{\mathrm{H}}\right)$. The amplitude and sign of the total theoretical EHE voltage depend both on the magnetization orientation at the center of each cross and the input current sense. In the following, we define the positive sense of the two input currents $\left(I_{1}\right.$ and $\left.I_{2}\right)$ as shown in Fig. 1(a) and define the positive pole of the output Hall voltage $\left(V_{\mathrm{H}}\right)$ at the left. The assignment of the input currents to the logic states is as follows: $-\mathrm{I}$ or $+\mathrm{I}$ correspond to the logic state " 0 " or" 1 " for both A and $\mathrm{B}$ crosses. The choice of the current amplitude I (in ampere) will affect $V_{H}$. The Hall voltage magnitudes of the two crosses are expected to be the same if the crosses have the same geometry. ${ }^{1-16}$ Thus, by defining the positive and negative maximum (zero) Hall signal as "1" ("0"), the XOR and XNOR gate functions are realized as the magnetizations of the two crosses being set antiparallel (Fig. 1(b)) and parallel (Fig. 1(c)), respectively. In the XOR gate where the magnetization of the left cross is set up, the input states " 0,0 " or " 1,1 " corresponding to the $(\uparrow \uparrow)$ or $(\downarrow \downarrow)$ input currents result in zero $V_{\mathrm{H}}$; while the input states " 0,1 " and " 1,0 " correspond to the $(\uparrow \downarrow)$ and $(\downarrow \uparrow)$ input currents result in a negative or positive maximum $V_{\mathrm{H}}$, respectively. As illustrated in Fig. 1, the logic XOR gate can be reconfigured to XNOR by switching the magnetic configuration of the two crosses from the antiparallel to parallel state. 

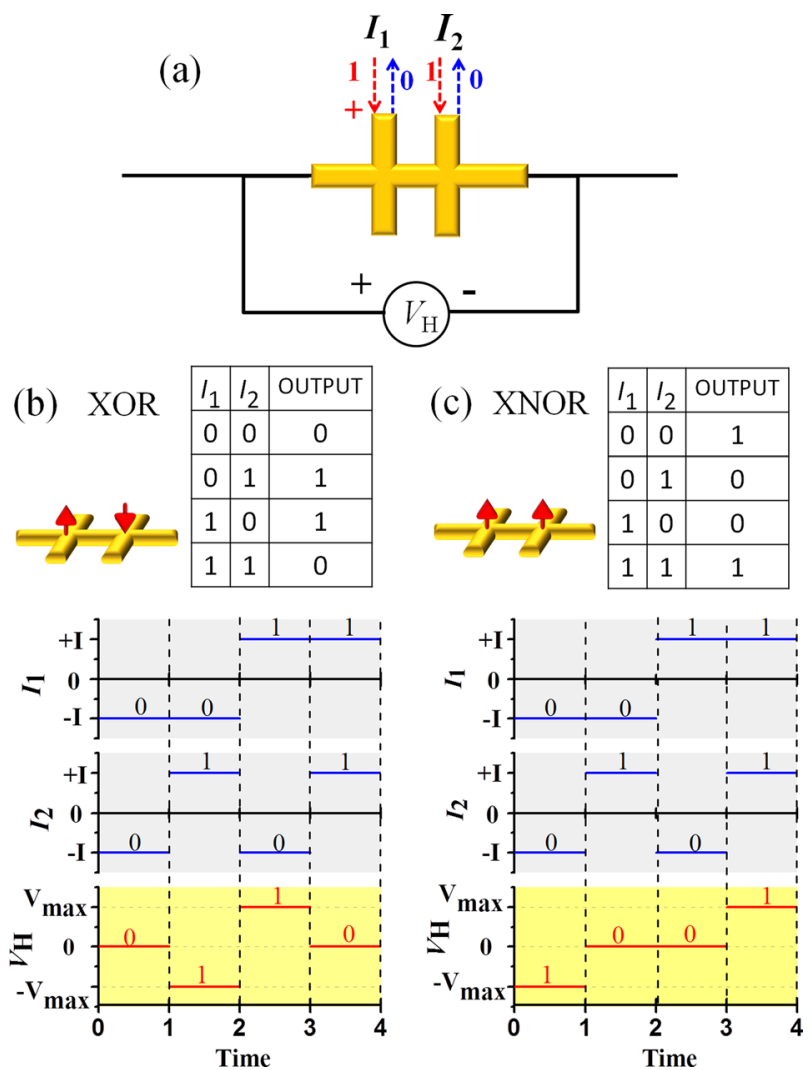

FIG. 1. (a) Schematics of the top view of the XOR or XNOR magnetic logic gate composed of two connected Hall crosses, where the sign of the field and the currents are defined. Theoretical logic states depending on the input currents are plotted in the picture; by defining the maximum (zero) Hall signal as $1(0)$, the (b) XOR and (c) XNOR gates are realized as the magnetization of the two nano-crosses being set antiparallel and parallel to each other, respectively.

As discussed in the introduction, similar logic devices can be used as an authentication system. Fig. 2(a) presents the architecture of the EHE-based authentication system we propose. For simplicity, only three EHE crosses are discussed here. The reference code is encoded in the Hall crosses by their magnetization orientations (up or down corresponds to logic state " 1 " or " 0 ," respectively). The user inputs are three currents $\left(I_{1}, I_{2}\right.$ and $\left.I_{3}\right)$ injected into the transverse arms of the three crosses with the definition of logic states as follows: - I (upward) or +I (downward) corresponds to logic " 0 " or " 1 ," respectively. The comparison between the user inputs and the reference codes is simply made by a Hall voltage measurement along the direction of the common longitudinal $\operatorname{arm}\left(V_{\mathrm{H}}\right.$, the positive pole is at the left of the common longitudinal arm). $V_{\mathrm{H}}$ is maximal $\left(+\mathrm{V}_{\max }\right)$ in one unique case and $+\mathrm{V}_{\max }$ read value indicates a match between the user input and the recorded code. Fig. 2(b) presents the theoretical results under all possible user inputs for a stored reference code " $1,0,1$." Here, $V_{\mathrm{H}}$ reaches its maximum only when the input is " $1,0,1$." With one, two or three mismatches, $V_{\mathrm{H}}$ decreases by step from $+\mathrm{V}_{\max }$ down to $-\mathrm{V}_{\max }$ value.

In order to demonstrate the feasibility of our EHE-based comparator device, we used $20 \mathrm{~nm}$ thick $\mathrm{Tb}_{16} \mathrm{Co}_{84}$ microand nano-structured films. TbCo alloy was chosen because it can hold high magnetic anisotropy ${ }^{18,19}$ and large Hall resistivity ${ }^{19,20}$ leading to both high thermal stability and high (a)
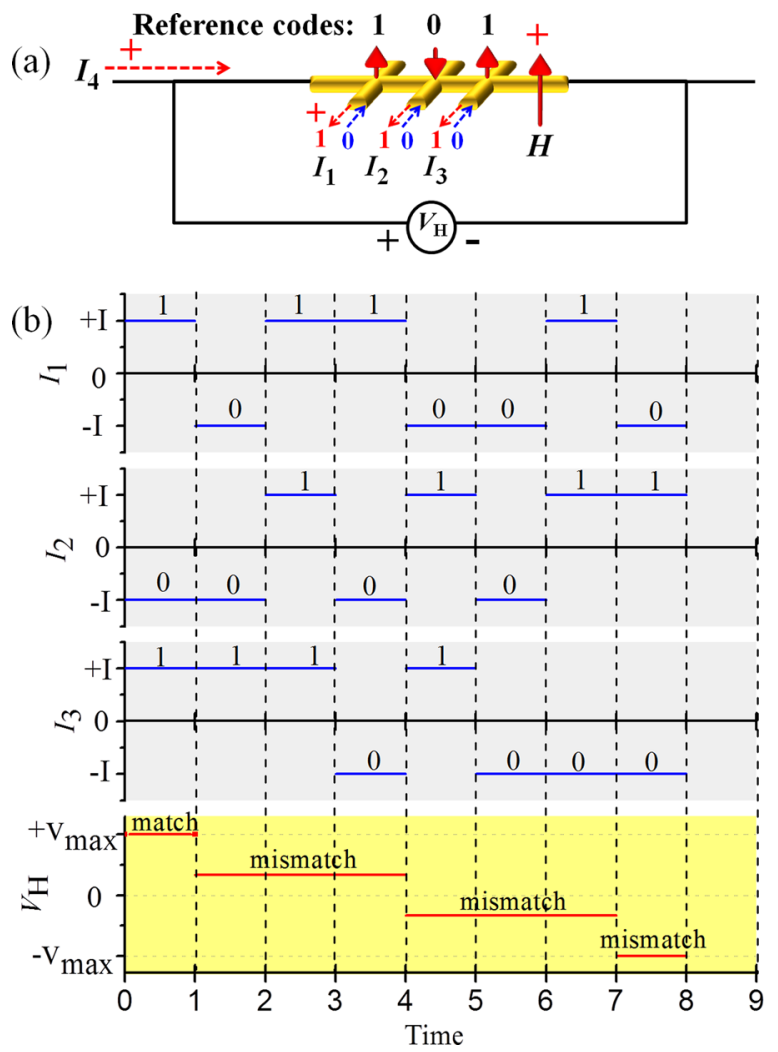

FIG. 2. (a) Schematics of the magnetic logic comparator composed of three connected Hall crosses. The sign of the field and currents are defined. The logic states assignment are plotted in the picture; (b) the theoretical results under all possible user inputs as the stored reference codes was "101" in (a). As shown, $V_{\mathrm{H}}$ reaches its maximum $\left(+\mathrm{V}_{\max }\right)$ only when the input also is " 101 ."

signal-to-noise ratio in devices, respectively. Moreover, TbCo coercivity can be modified by thermal excitation that is used here to write the reference code. We used $\mathrm{Ta}(3 \mathrm{~nm})$ as buffer layer to insure smooth surface to grow TbCo and Ta $(2 \mathrm{~nm}) / \mathrm{Pt}(3 \mathrm{~nm})$ bilayer as a cap layer to prevent oxidation. All layers have been grown by DC magnetron cosputtering on a Si substrate covered with a $100 \mathrm{~nm}$ thick thermal $\mathrm{SiO}_{2}$. The base-pressure was less than $5 \times 10^{-9}$ mbar in order to avoid materials oxidation. After film deposition, chains of crosses with width of $4 \mu \mathrm{m}$ or $300 \mathrm{~nm}$ were fabricated by combining optical lithography or electron beam lithography (EBL) and ion beam etching techniques. For magneto-transport measurements, the crosses were connected to external electrical sources via electrodes made by EBL and electron beam evaporation of $\mathrm{Ti}(10 \mathrm{~nm}) / \mathrm{Au}(50 \mathrm{~nm})$. The domain patterns of the $4 \mu \mathrm{m}$ crosses were characterized using magneto-optical Kerr microscopy, while the patterns of the $300 \mathrm{~nm}$ crosses were measured by magnetic force microscopy (MFM).

We used the following procedure, based on the specific magnetic properties of ferrimagnetic alloys, to write the magnetization direction of each Hall cross. Due to the distinct temperature dependence of the $\mathrm{Tb}$ and Co sublattice magnetizations and to their anti-ferromagnetic coupling, the overall magnetization (so-called net magnetization) is zero at a temperature called compensation temperature $\left(T_{\mathrm{CO}}\right){ }^{18,19}$ Consequently, the coercivity of $\mathrm{TbCo}$ increases as the net magnetization decreases and diverges when the net magnetization tends to zero. Then, the coercivity of TbCo film can 

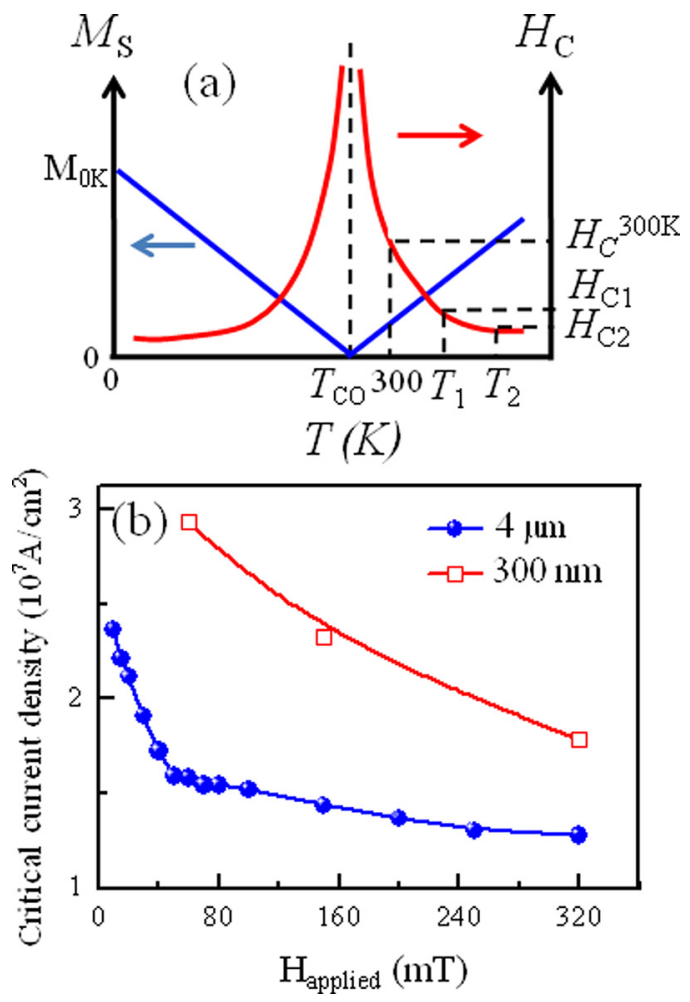

FIG. 3. (a) Typical behavior of the TbCo magnetization (blue) and coercivity (red) near the compensation temperature $\left(T_{\mathrm{CO}}\right)$. $\mathrm{Hc} 1$ and $\mathrm{Hc} 2$ correspond to coercive field at $\mathrm{T} 1$ and $\mathrm{T} 2$, respectively. (b) The minimum current density requested in the writing process for different applied magnetic field $H_{\text {applied }}$ and for two crosses sizes ( $4 \mu \mathrm{m}$ and $300 \mathrm{~nm}$ width). The red line is a guide for the eyes.

be easily tuned by adjusting temperature. Targeting a device working at room temperature (RT), the $\mathrm{Tb}_{16} \mathrm{Co}_{84}$ composition was chosen such that its $T_{\mathrm{CO}}$ is lower than RT so that the sample always remains in the Co-dominated regime, i.e., above $T_{\mathrm{CO}}$. The typical behaviors of its magnetization (blue) and coercivity (red) are reported in Fig. 3(a). Experimental data for similar TbCo alloy full film are presented for instance in Ref. 19. From the positive saturated state, an independent reversal of the magnetization of one cross is obtained through local heating. Currents of equal amplitude are simultaneously injected into two orthogonal arms of one cross (e.g., $I_{2}$ and $I_{4}$ for middle cross in Fig. 2(a)), such that the increase of temperature " $T_{2}-300 \mathrm{~K}$ " is much larger than in the other crosses, e.g., " $T_{1}-300 \mathrm{~K}$ " as shown in Fig. 3(a). Correspondingly, the coercivity in the selected cross $\left(H_{\mathrm{C} 2}\right)$ is much smaller than in the other crosses $\left(H_{\mathrm{C} 1}\right)$. Then, if an appropriate field $\mathrm{H}$ such that $H_{\mathrm{C} 2}<H<H_{\mathrm{C} 1}$ is applied while the currents are injected, the magnetization of the selected cross is reversed without affecting the neighboring crosses.

Kerr microscopy images in Figs. 4(a)-4(h), made on $4 \mu \mathrm{m}$ width TbCo nano-crosses, show all the eight possible domain patterns corresponding to the eight logic states that can be encoded using three crosses. The minimum current density needed to reverse the magnetization in one cross for different applied magnetic fields and for the $4 \mu \mathrm{m}$ wide crosses is summarized in Fig. 3(b). As expected, the reduction of current leads to a reduced temperature in the cross and so an increase of the applied field is required to reverse the cross magnetization (Fig. 3(a)). In our device, the typical current density is of the order of $10^{7} \mathrm{~A} / \mathrm{cm}^{2}$, comparable to values used to write data in STT SVs or MTJs. Note that we did not observe any domain wall displacement due to the STT effect in the present wire. The same writing approach is working nicely for both micronic and nanometric crosses. Fig. 4(i) shows an example of the written domain pattern measured by MFM in $300 \mathrm{~nm}$ wide crosses and Fig. 3(b) shows the minimum current density needed to reverse the magnetization in one cross for the $300 \mathrm{~nm}$ wide crosses. It is slightly higher than for the $4 \mu \mathrm{m}$ wire maybe because of a lower number of nucleation sites in the Hall crosses.

The eight logic states of the $4 \mu \mathrm{m}$ width TbCo crosses chain reported in Figs. 4(a)-4(h) are used to test the authentication system described in Fig. 2. The sign of fields and currents and the logic states assignment are the same as those used in Fig. 2(a). The Hall resistivity of all the nano-crosses was first checked by measuring $V_{\mathrm{H}}$ with an $\mathrm{I}=+100 \mu \mathrm{A}$ current injected into the transverse arms after the whole chain of crosses is uniformly magnetized up $\left(V_{\mathrm{H}}{ }^{\uparrow}\right)$ or down $\left(V_{\mathrm{H}}^{\downarrow}\right)$. The Hall resistivity $\left(\rho_{\mathrm{H}}\right)$ of the three nano-crosses is measured to be $1.675 \pm 0.025 \mu \Omega \cdot \mathrm{cm}$. The Hall resistivity variation from one cross to another is within 3\%. Table I shows experimental $V_{\mathrm{H}}$ values measured after the crosses were set as "1,0,1" ( $\downarrow \uparrow \uparrow)$ (cf. Fig. 4(g)) and the three input currents $\left(\mathrm{I}_{1}, \mathrm{I}_{2}, \mathrm{I}_{3}\right)$ are changed one by one. The maximum of Hall voltage $\left(\mathrm{V}_{\max }=0.17 \mathrm{mV}\right)$ is only reached for one current triplet (column 3 in Table I) due to the fixed $\uparrow \downarrow \uparrow$ crosses magnetic configuration. From this state, any change of current
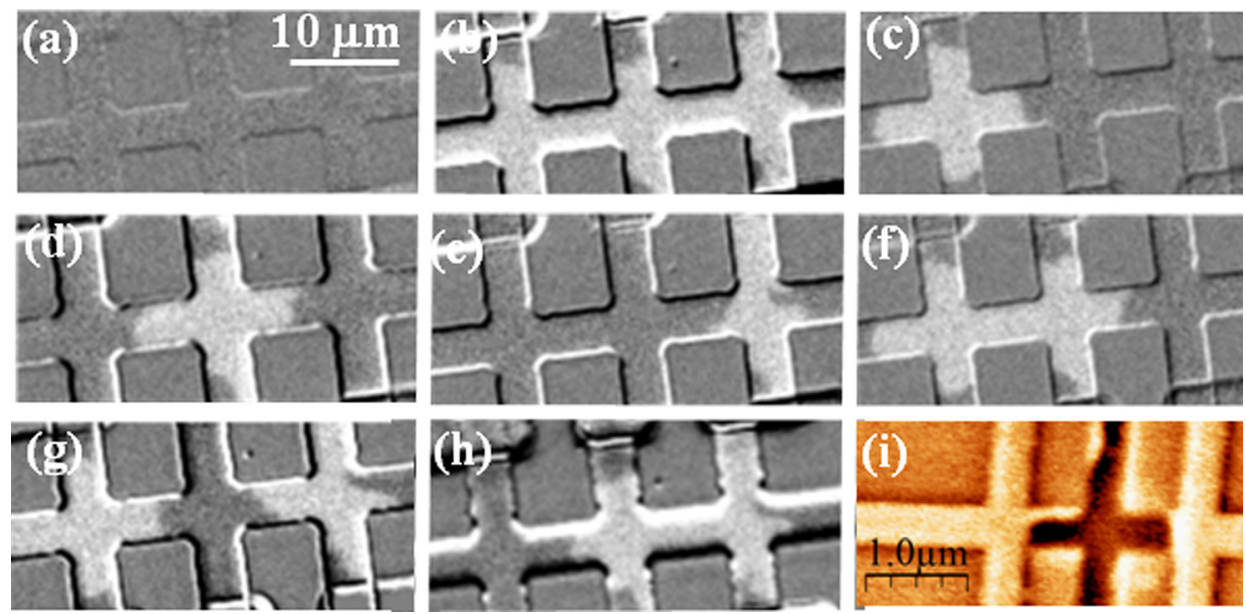

FIG. 4. (a)-(h) Kerr microscopy images of the eight possible domain patterns for the $4 \mu \mathrm{m}$ width TbCo nano-crosses. (i) MFM image of the $300 \mathrm{~nm}$ width nano-crosses chain with the domain pattern obtained using the same writing procedure. The white and black contrasts in the Kerr and MFM images correspond to magnetization pointing up and down, respectively. 
TABLE I. The $V_{\mathrm{H}}$ values measured after the moments of the crosses were set as $(\uparrow \downarrow \uparrow)$ (cf. Fig. $4(\mathrm{~g}))$ and the three input currents changed one by one. The current change in every step is underlined.

\begin{tabular}{lcccccc}
\hline \hline Step & 1 & 2 & 3 & 4 & 5 & 6 \\
\hline$I_{1}(\mathrm{~mA})$ & $+\underline{0.1}$ & +0.1 & +0.1 & +0.1 & +0.1 & $-\underline{0.1}$ \\
$I_{2}(\mathrm{~mA})$ & 0 & $-\underline{0.1}$ & -0.1 & -0.1 & $+\underline{0.1}$ & +0.1 \\
$I_{3}(\mathrm{~mA})$ & 0 & 0 & $+\underline{0.1}$ & $-\underline{0.1}$ & -0.1 & -0.1 \\
$V_{\mathrm{H}}\left(10^{-1} \mathrm{mV}\right)$ & 0.58 & 0.93 & 1.7 & 0.39 & -0.52 & -1.7 \\
\hline \hline
\end{tabular}

direction lowers $V_{\mathrm{H}}$. Table I consists of an experimental demonstration of the concept presented in Fig. 2. Such reading or comparison process is scalable down to nanometer width crosses as the Hall effect does not depend on the lateral dimension of the cross. Note finally that as shown in columns 1 and 2 of Table I, the maximum of Hall voltage can only be obtained if a current is simultaneously applied in the three Hall crosses.

In conclusion, based on the fact that the sign of extraordinary (EHE) voltage depends both on the magnetization orientation and the input current sense, we develop two original architectures of magnetic logic devices. The first one uses two crosses to form a logic gate with its function reconfigurable between XOR and XNOR as the magnetization orientation of the two crosses, respectively, switched between anti-parallel and parallel sates. The second device is a multi-bit logic comparator physically composed of a chain of crosses. This device can compare a user input with a reference encoded by the magnetization orientations of the cross cells. Comparing with similar concepts based on SVs or MTJs architectures, they possess simpler architecture, low cost fabrication process and are obtained in single magnetic layer. So they are promising for industrial implementation, especially for security and routers applications. The feasibility of both devices was proved on two prototypes made of $4 \mu \mathrm{m}$ and $300 \mathrm{~nm}$ width TbCo crosses chain. Although ferrimagnetic $\mathrm{TbCo}$ alloy may be very interesting because it provides reliable writing procedure and very good security level (if tuned to set $\mathrm{T}_{\mathrm{CO}}$ at room temperature), other materials and writing process may be investigated to improve EHE signal-to-noise ratio of the reading process as well as writing process energy consumption.

The authors acknowledge financial support from FEDER, the Region Lorraine, the Grand Nancy, and ANR COSMIQUE.

${ }^{1}$ B. Dieny, R. Sousa, S. Bandiera, M. Castro Souza, S. Auffret, B. Rodmacq, J. P. Nozieres, J. Herault, E. Gapihan, I. L. Prejbeanu, C. Ducruet, C. Portemont, K. Mackay, and B. Cambou, IEEE Int. Electron Devices Meet. 2011, 1.3.1 (2011).

${ }^{2}$ A. Ney, C. Pampuch, R. Koch, and K. H. Ploog, Nature 425, 485 (2003).

${ }^{3}$ S. Datta, S. Salahuddin, and B. Behin-Aein, Appl. Phys. Lett. 101, 252411 (2012).

${ }^{4}$ C. Sanid and S. Murugesh, Jpn. J. Appl. Phys., Part 1 51, 063001 (2012).

${ }^{5}$ T. Kagami, T. Kuwashima, S. Miura, T. Uesugi, K. Barada, N. Ohta, N. Kasahara, K. Sato, T. Kanaya, H. Kiyono, N. Hachisuka, S. Saruki, K. Inage, N. Takahashi, and K. Terunuma, IEEE Trans. Magn. 42, 93 (2006). ${ }^{6}$ J. G. Zhu and C. Park, Mater. Today 9(11), 36-45 (2006).

${ }^{7}$ Y. Huai, F. Albert, P. Nguyen, M. Pakala, and T. Valet, Appl. Phys. Lett. 84, 3118 (2004).

${ }^{8}$ J. G. Zhu, Y. F. Zheng, and G. A. Prinz, J. Appl. Phys. 87, 6668 (2000).

${ }^{9}$ R. C. Sousa and I. L. Prejbeanu, C. R. Phys. 6, 1013 (2005).

${ }^{10}$ J. A. Katine and E. E. Fullerton, J. Magn. Magn. Mater. 320, 1217 (2008).

${ }^{11}$ S. I. Kiselev, J. C. Sankey, I. N. Krivorotov, N. C. Emley, R. J. Schoelkopf, R. A. Buhrman, and D. C. Ralph, Nature 425, 380 (2003).

${ }^{12}$ Z. M. Zeng, P. Upadhyaya, P. Khalili Amiri, K. H. Cheung, J. A. Katine, J. Langer, K. L. Wang, and H. W. Jiang, Appl. Phys. Lett. 99, 032503 (2011).

${ }^{13}$ S. Ikeda, K. Miura, H. Yamamoto, K. Mizunuma, H. D. Gan, M. Endo, S. Kanai, J. Hayakawa, F. Matsukura, and H. Ohno, Nat. Mater. 9, 721 (2010).

${ }^{14}$ A. W. Smith and R. W. Sears, Phys. Rev. 34, 1466 (1929).

${ }^{15}$ R. Karplus and J. M. Luttinger, Phys. Rev. 95, 1154 (1954).

${ }^{16}$ J. Moritz, B. Rodmacq, S. Auffret, and B. Dieny, J. Phys. D: Appl. Phys. 41, 135001 (2008).

${ }^{17}$ S. L. Zhang, Y. Liu, L. J. Collins-McIntyre, T. Hesjedal, J. Y. Zhang, S. G. Wang, and G. H. Yu, Sci. Rep. 3, 2087 (2013).

${ }^{18}$ P. Hansen, C. Clausen, G. Much, M. Rosenkranz, and K. Witter, J. Appl. Phys. 66, 756 (1989).

${ }^{19}$ M. Gottwald, M. Hehn, F. Montaigne, D. Lacour, G. Lengaigne, S. Suire, and S. Mangin, J. Appl. Phys. 111, 083904 (2012).

${ }^{20}$ T. W. Kim and R. J. Gambino, J. Appl. Phys. 87, 1869 (2000). 